\title{
La société européene: les enjeux et les perspectives
}

L'Europe ne se fera pas en un jour, ni sans heurts. Rien de durable ne s'accomplit dans la facilité. Pourtant elle est déjà en marche. Cette idée

"Europe » sera la force contre laquelle se briseront tous les obstacles.

Robert Schumann

\section{Introduction : l'origine de la Société Européenne}

Depuis octobre 2004, les entreprises disposent d'une option supplémentaire quant au choix de leur régime juridique dans le cadre duquel elles souhaient conduire leurs activités européennes. L'abréviation « SE » correspond au nom latin (officiel) de la société européenne, Societas Europea. Le principal objectif de la SE est de permettre aux entreprises de mener leurs activités par-delà les frontières européennes dans un même cadre juridique ${ }^{1}$.

Le statut de la Societas Europea est régi par deux textes de droit européen en date du 8 octobre 2001: le règlement $\mathrm{CE} \mathrm{n} 2157 / 2001^{2}$, qui organise son statut de droit commercial et la directive $\mathrm{CE} \mathrm{n}^{\circ} 2001 / 86^{3}$, qui définit, au plan du droit social, les conditions de l'implication des salariés nécessairement attachées à ce statut. À ces deux textes qui concernent spécifiquement la SE, il faut ajouter la directive du 17 février 2005 modifiant la directive du 23 juillet 1990 portant sur « un régime fiscal commun applicable aux fusions, scissions, apports d'actifs et échanges d'actions intéressant des sociétés d'Etats membres diffé-

1 M. Stollt, E. Wolters, Implication des travailleurs dans la Société européenne (SE). Guide pour les acteurs de terrain, Bruxelles 2013, p.70.

2 Règlement CE n²157/2001 du Conseil du 8 octobre 2001, relatif au statut de la société européenne, (J.O.L 294 du 10 novembre 2001).

3 Directive CE n²001/86 du Conseil, du 8 octobre 2001, complétant le statut de la société européenne pour ce qui concerne l'implication des salariés, (J.O.L 294 du 10 novembre 2001). 
rents ", visant toutes les sociétés de capitaux ${ }^{4}$, modifiée par la directive 2005/19/CE du Conseil du 17 février 2005 modifiant la directive 90/434/CEE ${ }^{5}$ et la directive 2006/98/ $\mathrm{CE}$ du Conseil du 20 novembre 2006 portant adaptation de certaines directives dans le domaine fiscal, en raison de l'adhésion de la Bulgarie et de la Roumanie ${ }^{6}$. Cette derniere directive consacre la « neutralité fiscale » des opérations transnationales menées en Europe, que la société concernée décide de transférer son siège d'un Etat à l'autre.Visant toutes les sociétés de capitaux, ce texte a vocation à s'appliquer plus particulièrement aux $\mathrm{SE}$ en raison des facilités de mobilité intra-européenne que leur offre leur statut.

La SE n'est pas la première forme juridique européenne créée en faveur des entreprises. Le premier instrument juridique de rapprochement à l'échelle europénne fut le GEIE7. Il s'agit d'un regroupement de personnes morales (sociétés ou autres entités juridiques), de droit privé ou public, décidant de mettre en commun des moyens tout en conservant leur personnalité juridique propre. Un GEIE doit compter au minimum deux sociétés européennes appartenant à deux États membres différents. Son siège doit être obligatoirement fixé sur le territoire de l'Union et de l'Espace économique européen. L'Espace économique européen (EEE) est une union économique rassemblant trente Etats européens : les vingt-huit États membres de l'Union européenne (UE), moins la Croatie, et trois des quatre États membres de l'Association européenne de libre-échange (AELE). Jusqu'au 1er janvier 2009, l' Union européenne n'était pas directement partie l'accord : elle l'était indirectement par la Communauté européenne (CE), l'un des piliers composant l'Union européenne. Depuis la disparition de la structure de l'Union en piliers, l'UE en tant que telle est membre de l'EEE. Il ne peut employer plus de 500 personnes. Le GEIE, n'étant pas une société, n'a pas obligatoirement un capital minimal et n'a pas pour objectif de réaliser des bénéfices pour lui-même. Ses bénéfices éventuels sont répartis entre ses membres, selon les clauses du contrat d'association, en principe au prorata des moyens engagés par chacun, et taxés selon les règles applicables à ces sociétés. Les membres du GEIE sont indéfiniment et solidairement responsables des dettes contractées par celui-ci. Il est vraie que le groupement peut être qualifié de "structure d'appoint ", destiné à la coopération entre ses membres alors que la SE a été créée dans une souci de concentration. Cette structure a été inspiré du modèle français, le GEIE en

4 Directive 90/434/CEE du Conseil, du 23 juillet 1990, pour un régime fiscal commun appliacable aux fusions, scissions, apports d'actifs et échanges d'actions intéressant des sociétés d'Etats membres différents, (J.O.L 225 du 20 août 1990).

5 J.O.L 58 du 4 mars 2005.

6 J.O.L 363 du 20 décembre 2006.

7 Un groupement européen d'intérêt économique (GEIE) est une entité juridique fondée sur le droit européen. C'est l'adaptation au cadre international européen du concept français de Groupement d'intérêt économique (GIE). Il a été institué par le Règlement CEE n 2137/85 du Conseil du 25 juillet 1985. Son objectif est de faciliter la coopération transnationale entre entreprises. 
vigueur dès 1967 qui avait pour objectif de permettre aux chefs d'entreprises de mettre en communs leurs moyens de commercialisation, tout en restant juridiquement indépendants. Cette réglementation souple et novatrice intéressa la Commision qui décida de lancer des travaux, parallèlement aux réflexions sur la société européenne.

L'idée de créer des sociétés de statut européen, bénéficiant d'une liberté de circulation sur le marché européen comparable à celle dont les sociétés de droit local jouissent sur leur marché domestique, est ancienne. C'est en effet dès 1949 qu'au sein du Conseil de l'Europe fut envisagé un statut de « compagnies européennes » censé contribuer, à travers le regroupement des facteurs de production, à la reconstruction économique d'un continent qui ne s'était pas totalement relevé de la guerre. Le projet de créer un statut de société européenne, en mesure de se déployer librement à l'échelle du marché unique, a été repris après 1958. Le projet a toutefois été mis en sommeil jusqu'à un mémorandum présenté par le gouvernement français en 1965, incitant la Commission européenne à s'emparer véritablement du sujet ${ }^{8}$. Un groupe d'experts, juristes de haut niveau, est alors chargé d'élaborer un texte. Le rapport de ce groupe, présidé par le professeur Sanders, est rendu public en 1967. Il marque un tournant. Pour la première fois, le statut de la SE faisait l'objet de propositions aussi précises qu'innovantes. Aussi la Commission suivit-elle la plupart des suggestions du rapport Sanders qui sont reflétées dans ses premières propositions de règlements de 1970 puis de $1975^{9}$.

\section{La rôle de la France}

La législation française subit l'influence de l'Union Européenne par l'adoption de directives portant sur le droit des sociétés. Le traité de Rome modifié tend à assurer la liberté d'établissement, en supprimant les obstacles à cette liberté contenus dans les législations des États-membres. C'est pourquoi, l'essentiel des efforts normatifs des autorités européennes en matière de droit des sociétés consiste à garantir l'équilibre entre les sociétés tout en protégeant les intérêts des tiers et des associés ${ }^{10}$.

Depuis 1968, plus de douze directives ont été adoptés pour tendre vers une harmonisation du droit européen des sociétés. Le droit de l'Union est incontestablement une source importante de réglementation des sociétés. Il faut toutefois noter qu'il touche

$8 \mathrm{La}$ France a joué un rôle actif dans la relance du projet de la SE, que ce soit par le biais d'initiatives privées- congrès des notaires de Tours 1959 ou gouvernementales. Mémorandum du 15 mars 1965 incitant la Commission à former un groupe d'experts chargé d'élaborer un avant-projet de status de la SE. C'est enfin sous les auspices de la présidence française, lors du Conseil européen de Nice de décembre 2000, qu'a été trouvé l'accord politique sur la SE.

9 « Proposition de règlement $\mathrm{CEE}$ du Conseil portant statut de la société anonyme européenne ", (J.O.C.E., 10 octobre 1970, n 124).

10 J.P. Bertrel, La société européenne entre son passé et son avenir, " Droit \& patrimoine ", n¹25, avril 2004, p. 46. 
surtout aux sociétés de capitaux, c'est-à-dire aux grandes sociétés et aux sociétés cotées en particulier. Dans ce dernier secteur, le droit européen est particulièrement prolixe ; les sociétés disposant de la liberté d'établissement, les textes de droit européen visent à la reconnaissance mutuelle des sociétés et des personnes morales ${ }^{11}$. Afin de développer les échanges intra européens, une proposition de règlement a vu le jour portant sur le statut de société anonyme européenne dès 1970. Cette proposition était favorable à la constitution d'une société régie entièrement par un droit autonome directement applicable dans tous les états membres. Plus modestement, il fut donc proposé un règlement le 25 juillet 1985 portant sur le Groupement Européen d'Intérêts Economique destiné à faciliter l'activité économique. Aujourd'hui on le retrouve dans l'article L. 252-1 Code de commerce.

La société europénne a finalement abouti le 20 décembre 2000 au sommet de Nice et a été adoptée le même jour par un règlement sur les statuts européens qui est devenu le règlement du 8 octobre 2001 et une proposition de directive qui concerne la situation des travailleurs. Ces textes on été transposés par la loi «Breton » en 200512. La Loi « Breton » adapte et transpose en droit français le régime de la société européenne institué par le Règlement Européen CE n ${ }^{\circ}$ 157/2001 du 8 octobre 2001 en introduisant des règles nationales dans le Code de commerce et dans le Code du Travail. Les sociétés européennes immatriculées en France seront donc régies en premier lieu par le Règlement Européen, et pour les matières non réglées par le Règlement, par les nouveaux articles du Code de Commerce et du Code du Travail et par les règles applicables aux sociétés anonymes non contraires à ceux-ci et enfin par leurs dispositions statutaires. L'organisation de la $\mathrm{SE}$ immatriculée en France est très proche de celle de la SA avec toutefois des règles dérogatoires, principalement en ce qui concerne les relations entre actionnaires. Elle offre pour les sociétés de droit français des facultés nouvelles attachées à la qualité de société européenne, notamment le transfert de siège à l'étranger, la fusion internationale, etc... La Loi « Breton » contient deux volets : un volet droit des sociétés qui précise les règles de constitution et fonctionnement des SE ayant leur siège en France et un volet droit social qui concerne l'implication des salariés (en application de la directive CE $n^{\circ}$ 2001/86 du 8 octobre 2001). Toutefois, l'application de certaines dispositions importantes reste subordonnée à la parution d'un décret (notamment règles de publicité) ${ }^{13}$.

La Loi « Breton » précise le régime selon lequel la SE peut être constituée en France, c"est-à-dire :

11 Ibidem, p.53.

12 Un chapitre IX a été introduit dans le Code de commerce aux articles L. 229-1 et suivantes.

13 J.P. Dom, La société européenne - Aspects droit des sociétés, " Droit \& Patrimoine ", Avril 2004, $\mathrm{n}^{\circ} 125$, p. 65. 
par voie de fusion ${ }^{14}$;

par constitution d'une SE holding ${ }^{15}$;

par création d'une filiale ;

par voie de transformation ${ }^{16}$.

\section{La création de la SE}

La SE ne peut être créée directement par des personnes physiques. Et les sociétés à la base de sa création doivent en outre être des personnes morales ayant déjà une dimension transnationale européenne ${ }^{17}$. Lorsque la SE est créée par voie de fusion, les sociétés participant à l'opération doivent être implantées dans au moins deux Etats membres différents. Pour pouvoir constituer une SE holding ou une SE filiale, elles doivent soit relever du droit d'Etats membres différents, soit posséder au moins une filiale ou une succursale implantée dans un Etat membre autre que celui de leur siège statutaire et ce, depuis deux ans au moins ; la filiale devant relever du droit d'un autre Etat membre. Comme le fait remarquer le professeur Michel Menjucq, la SE est : " la société de sociétés, car elle peut n'avoir que des actionnaires personnes physiques lors de son immatriculation, par exemple, en cas de constitution d'une SE holding; toutefois, même dans ce cas, des personnes morales ayant en l'occurence la forme de société anonyme et/ou de société à responsabilité limitée, doivent être à l'iniatitive du projet de constitution en promouvant l'opération ${ }^{18}$. La SE bénéficie a priori en quelque sorte d'une "préférence européenne ». Si les Etats n'en décident pas autrement, en effet, seules les sociétés ayant leur siège statutaire dans l'Union peuvent participer à sa création $^{19}$. Dans le cas où les Etats optent pour cette possibilité, encore faut-il cependant que la société concourant à la constitution de la SE ait « un lien effectif et continu avec l'économie d'un Etat membre $»^{20}$.

La création d'une SE holding est ouverte aux sociétés anonymes et aux sociétés à responsabilité limitée. Les sociétés participantes doivent adopter un projet de constitution qui fixe la pourcentage minimal des actions ou parts de chacune des sociétés promeuvant

14 Délivrance par le greffe d'un certificat attestant de l'accomplissemen des formalités et contrôle de la légalité de la procédure de fusion par un notaire.

15 Dépôt par les sociétés françaises créant la SE d'un projet de constitution au greffe, application des règles de protection des fusions aux créanciers des sociétés françaises créant la SE (droit d'opposition, approbation de la masse des obligataires).

16 Dépôt au greffe du projet de transformation d'une SA française en SE, rapport du commissaire à la transformation sur les capitaux propres.

17 Article 2 du règlement du Conseil CE n 2157/2001.

18 N. Lenoir, L'avenir de la "Societas Europea », " Journal de droit europeen », Février 2008, p. 68.

19 Article 2.5 du règlement du Conseil CE n² 2157/2001.

20 Un cadre réglementaire moderne pour le droit européen des sociétés. Rapport du groupe de haut niveau d'experts en droit des sociétes, Bruxelles 2002, p. 57. 
l'opération que les actionnaires devront apporter pour que la SE soit constituée. Ce pourcentage doit consister en actions conférant plus de 50\% des droits de vote permanents. Ce projet est déposé et publié par une procédure similaire à celle des fusions. C'est à l'assemblée générale de chacune des sociétés participantes d'approuver le projet ${ }^{21}$. Toutefois, à la différence de la procédure de fusion, cette approbation ne suffit pas. Le règlement du Conseil CE n² 2157/2001 remet logiquement la décision finale entre les mains des actionnaires ou des porteurs de parts des sociétés. Ils ont à faire connaître dans les trois mois s'ils ont l'intention d'apporter leurs titres. Ce n'est que si, dans ce délai, le pourcentage d'apports de titres requis a été atteint que la SE holding peut être constituée ${ }^{22}$. Les sociétés participantes, à la difference de la fusion, ne disparaissent pas, mais deviennent totalement ou majoritairement contrôlées par la SE holding. Par analogie avec la fusion, un Etat membre peut prévoir, en ce qui concerne les sociétés qui promeuvent l'opération, une protection renforcée des associés minoritaires opposés à l'opération, des créanciers et des salariés alors même que les actionnaires ne peuvent être contraints d'échanger leurs titres et que les sociétés participantes ne disparaissent pas. La SE holding (que ses actions soient ou non cotées), en garantissant l'unité de direction d'un groupe et en permettant aux associés de mieux structurer leurs pouvoirs, répond à la vocation de la SE. Pour autant, la procédure de création d'une SE holding semble peu utilisée. Parmi les raisons avancées pour expliquer le manque d'attractivité de de régime juridique, figure l'exigence de la présentation par les dirigeants des sociétés promotrices d'un rapport expliquant et justifiant les aspects juridiques et économiques de la constitution et indiquant les conséquences pour les actionnaires et les salariés de l'adoption de la forme de SE. Cette contrainte semble inadaptée lorsque les dirigeants des sociétés participantes ont besoin de conserver sur leur stratégie une certaine confidentialitée ${ }^{3}$. Cela est surtout vraie pour les holdings dits « de rachat " pratiquant le LBO, à savoir "l'opération de rachat d'entreprise en finançant une part importante du prix d'acquisition par endettement ». Une société holding qui s'endette est constituée pour acheter une entreprise dont les excédents de trésorie remonteront régulièrement au niveau du holding via des dividendes afin de lui permettre de payer les intérêts de sa dette et de la rembourser ${ }^{24}$. Cependant, au lieu de

21 F.Mary, Ch.A. Guelluy, Fusions transfrontalières: le coup d'envoi ?, « Option Finance », n 1018, Mars 2009, p. 74.

22 Article 3.3 du règlement du Conseil CE n² 2157/2001.

23 A.S. Cornette de Saint-Cyr, O. Rault, Aspects juridiques et socoaux des opérations de fusions au sein de l'Union européenne, JCP/La semaine juridique- Édition entreprises et affaires, $\mathrm{n}^{\circ} 15$, Avril 2008, p. 143.

24 Le LBO (Leverage Buy Out / Opération de rachat d'une société avec l'effet de levier), s'est peu à peu imposé comme une nouvelle technique de financement et est aujourd'hui largement pratiqué. Le LBO est un mécanisme qui fait intervenir une personne morale agissant en tant que holding (souvent une $S A S$ ) créée à cet effet. Il s'agit de racheter la cible en utilisant un endettement bancaire contracté par la holding. Ainsi il devient possible de prendre le contrôle 
racheter directement lsa participation, cela se fait par le biais d'une holding. Cette holding sera constituée de deux types d'associés : l'initiateur qui a pour dessein d'acquérir cette participation et les investisseurs dont l'intervention est uniquement guidée par des fins spéculatives. Ces derniers sortant d'ailleurs du montage quand celui-ci arrive à son terme. La holding a donc pour fonction de réaliser cette prise de contrôle.

Les dispositions du règlement du Conseil $\mathrm{CE} \mathrm{n}$ n $^{2}$ 2157/2001 sur la création d'une SE filiale sont d'une grande sobriété et d'une grande souplesse. La procédure est d'abord ouverte, non pas seulement aux SA, voire aux SARL, mais aussi à n'importe quelle entité juridique de droit public ou privé en Europe. Malgré ces conditions favorables, la $\mathrm{SE}$ filiale ne rencontre à ce jour pas plus de succès que la SE holding. Il existerait six $\mathrm{SE}$ constituées en tant que filiales, dont aucune n'est cotée en bourse ${ }^{25}$. À titre d'exemple, Eurotunnel SE, immatriculée en Belgique, est destinée à améliorer la coordination des opérations commerciales entre les filiales existantes d'Eurotunnel actuellement présentes au Royaume-Uni, France, Benelux, Allemagne et Espagne et ses activités dans la commercialisation de services de fret. Cette nouvelle filiale devrait à terme intégrer toutes cs activités, dans un but de simplification de la structure du groupe et de la réalisation d'économies ${ }^{26}$.Le Groupe Eurotunnel gère et commercialise directement un service de transport par navettes via le Tunnel sous la Manche transportant des passagers et des véhicules de transport de marchandises. Il gère également le passage de trains à très grande vitesse (Eurostar) et les services de fret ferroviaire d'autres compagnies ferroviaires via le Tunnel sous la Manche. Le Groupe gère aussi la filiale Europorte (qui comprend la société anglaise GB Railfreight) qui propose une large gamme de services de fret ferroviaire intégrés à la fois en France et au Royaume-Uni.

Peu utilisée, la formule de la SE filiale mériterait de susciter devantage d'intérêt. Elle peut être utile notamment pour mettre en place des entreprises communes coopératives tout en aidant à surmonter les problèmes éventuels liés à la préservation d'un équilibre entre les nationalités des sociétés mères. La SE filiale devrait aussi constituer à terme l'un des cadres privilégiés de la coopération entre le public et le privé. Un centre de recherche universitaire et une industrie pharmaceutique peuvent avoir avantage à constituer une SE filiale pour développer des recherches ; structure plus efficace qu'un $\mathrm{GEIE}^{27}$. Celui-ci ne peut faire de bénéfices, contrairement à la SE filiale dont l'activité peut, par exemple,

d'une société en minimisant son apport personnel. Le LBO a pour objet de prendre le contrôle d'une société cible.

25 Latham\&Watkins, Quelles nouvelles mesures pour le droit des sociétés et le droit boursier ?, "Panorama législatif été 2005 ", Quelles nouvelles mesures pour le droit des sociétés et le droit boursier?, Paris 2005, p. 87.

26 Communiqué de presse du groupe Eurotunnel « Eurotunnel to set up a services company under European Law : « Eurotunnel SE », 30 mars 2006 et http://bourse.latribune.fr/actions/ nyse-euronext-paris/groupe-eurotunnel/societe-activites-perspectives-20084270.html.

27 Ibidem, p. 92. 
être d'exploîter des brevets. Les entreprises devraient trouver dans la formule de la SE filiale un autre intérêt lié à la possibilité offerte à une SE de constituer elle-même une ou plusieurs SE filiale unipersonnelles. Alors que dans les Etats membres, la SA unipersonnelle n'existe en général pas (certains Etats, comme l'Autriche, en incorporant le statut de la $S E$ dans leur législation en ont profité pour créer un statut de SA unipersonnelle). Une SE pourra alors se doter d'une chaîne de SE unipersonnelles à la gouvernance partiellement harmonisée avec celles de la SE mère. Les SE unipersonnelles sont soumises aux dispositions nationales adoptées conformément à la directive du 21 décembre 1989 sur les SARL unipersonnelles ${ }^{28}$.

La transformation en SE est réservée aux seules SA possédant depuis deux ans au moins une filiale dans un Etat membre autre que celui de leur siège ; ce qui exclut les succursales. En dépit de cette condition restrictive, cette procédure a l'avantage de la simplicité. Contrairement à l'hypothèse de sa fusion transfrontalière, la transformation évite l'application cumulative ou distributive de législations nationales différentes ${ }^{29}$. D'un point de vue fiscal l'opération en outre est neutre. Les modalités de transformation d'une SA en SE sont inspirées de procédures de création d'une SE par voie de fusion ou d'une SE holding, mais, le règlement du Conseil CE n² 2157/2001 renvoie plus largement encore au droit national qu'en matière de fusion. Il se borne à prescrire un projet de transformation (sans en préciser le contenu) assorti du rapport, expliquant et justifiant l'opération, à présenter par les organes dirigeants, ainsi qu'un rapport d'expertise attestant que " la société dispose d'actifs, nets au moins équivalents au capital augmenté des réserves que la loi ou les statuts ne permettent pas de distribuer $»^{30}$. Le projet de transformation et les statuts de la SE sont adoptés, comme dans les autres cas de création d'une $\mathrm{SE}$, par l'assemblée générale extraordinaire à la majorité qualifiée. Les textes, aussi bien européens que français, conduisent à retenir une interprétation selon laquelle la transformation d'une SE ne peut se réaliser qu'en SA. En effet, l'article 66.1 du règlement du Conseil $\mathrm{CE} \mathrm{n}{ }^{\circ}$ 2157/2001 se réfère expressément à la transformation de la $\mathrm{SE}$ en « société anonyme relevant du droit de l'Etat membre de son siège statutaire " ${ }^{31}$. En écho à cet article, l'article L. 229-10 du Code de Commerce n'envisage la transformation de la SE qu'en société anonyme. Il est très probable que la question de la transformation de la $\mathrm{SE}$ en SAS n'a effleuré ni l'esprit du législateur européen, ni celui du législateur français. On ne peut que le regretter et appeler de nos voeux qu'une telle transformation soit rendue

28 Douzième directive du Conseil n 89/667/CCE, du 21 décembre 1989, en matière de droit des sociétés concernant les sociétés unipersonnelles à responsabilité limitée, (J.O.L 395 du 30 décembre 1989).

$29 \mathrm{G}$. Lesieur, Le nouveau régime légal des fusions tranfrontalières issu de la directive communautaire du 26 octobre 2005, « Les Petites Affiches ", Août 2008, p.4 et suivantes.

30 Article 37 du règlement du Conseil $\mathrm{CE} \mathrm{n}^{\circ}$ 2157/2001.

$31 \mathrm{G}$. Lesieur, Le nouveau régime légal des fusions tranfrontalières issu de la directive européenne du 26 octobre 2005, "Les Petites Affiches ", Août 2008, p.8. 
possible. Si tel devait être le cas, il conviendrait d'adapter le cadre textuel aux singularités de la SAS, notamment d'imposer que cette transformation intervienne à l'unanimités2.

\section{La gestion de la SE}

En Europe, il existe deux systèmes de gouvernance d'entreprise: le système moniste avec conseil d'administration unique assurant la gestion de l'entreprise et le système dualiste avec, d'une part, un organe de direction/directoire responsable de la gestion courante des activités et, d'autre part, un conseil de surveillance chargé de contrôler l'organe de direction/directoire. Jusqu'à présent, le choix de la structure dépendait essentiellement des dispositions légales du pays. Seuls quelques Etats membres permettaient déjà aux entreprises de choisir librement entre le modèle moniste et modèle dualiste (par exemple, France, Finlande, Hongrie et Italie). Une SE peut opter librement pour un système moniste ou dualiste, quelque soit le pays dans lequel elle est implantée. Cette règle vaut également pour les pays où ce choix n'existe pas pour les régimes juridiques nationaux.

$\mathrm{Au}$ niveau des organes de gestion et de direction, le règlement du Conseil $\mathrm{CE} \mathrm{n}^{\circ}$ 2157/2001 prévoit, selon le choix des actionnaires, deux types de structures : le monisme avec un conseil d'administration et le dualisme avec conseil de surveillance et directoire. Le droit commercial français connaissait déjà ces deux systèmes et n'a donc pas eu à faire l'objet de modifications sur ce point. En Belgique, et au Royaume-Uni, où seul le monisme était connu, des dispositions spécifiques à la $\mathrm{SE}$ permettent d'opter pour un régime dualiste ${ }^{33}$. Parallèment, en Allemagne, seule la loi sur les SE permet d'opter pour le régime moniste, alors que le droit commun des SA ne prévoit encore que le dualisme. En dehors de ce cadre optionnel entre dualisme et monisme, le règlement du Conseil CE n²157/2001 renvoie aux législations nationales ou aux statuts (éventuellement encadrés par ces législations) le soin de déterminer les règles de fonctionnement des organes dirigeants de la SE. Ces renvois concernent aussi bien la nomination et le nombre des membres de ces organes que leur révocation, leurs fonctions et leurs rôles, la périodicité de leurs réunions, les processus de délibération quant aux quorum notamment, ou encore l'information à fournir aux membres de conseils ${ }^{34}$. Peu de dispositions s'appliquent de manière impérative sans pouvoir être modifiées ou aménagées au niveau du droit local ou par les statuts, et celle suivant laquelle l'organe d'administration (dans le système moniste) élit d'un président parmi les membres désignées. Ce président des organes soit un représentant des actionnaires et non pas des salariés.

32 Cozian M., Deboissy F.,Viandier A., Droits des sociétés, Paris 2010, p.267.

33 N. Lenoir, L'avenir de la "Societas Europea », " Journal de droit europeen », Février 2008, p. 78. 34 Ibidem, p. 84. 
Le plus souvent, le règlement de 2001 propose une harmonisation minimale. C'est le cas en ce qui concerne le nombre des organes de la $\mathrm{SE}^{35}$. Parfois, le règlement prévoit une simple option, comme celle qui permet de déléguer la gestion courante de la société, dans les mêmes conditions que pour les SA de droit local, à un ou plusieurs directeurs généraux ${ }^{36}$. Cette disposition, qui a pour objet de délimiter les responsabilités au sein des organes de la société, n'existe pas dans tous les droits nationaux, comme en France et en Belgique où cette option a été levée. Autre cas de figure, le règlement renvoie directement ou indirectement aux statuts. Par exemple, concernant le système dualiste, il renvoie aux statuts le soin de fixer le nombre des organes de surveillance et de direction. De même, s'il prévoit que les membres du directoire, dans le système dualiste, sont nommés et révoqués par le conseil de surveillance, il laisse aux Etats la liberté statutaire de conférer ces pouvoirs à l'assemblée générale. La multiplication de ces renvois, options et exceptions réduit la lisibilité du dispositif de gouvernance de la SE en le faisant par trop dépendre du lieu d'implantation du siège statutaire. Le règlement du Conseil CE n²157/2001 fait ressortir des lignes de la force qui forment la colonne vertébrale du statut de la SE. D'abord, il y manque le souci de garantir une séparation des pouvoirs entre organes de la société : conseil de surveillance et directoire, conseil d'administration et directeurs généraux. Ensuite, il se réfêre au droit à l'information des membres des organes et à leur devoir de confidentialité clairement érigés en principes de bonne gouvernance ${ }^{37}$. Enfin, les modes de nomination et de révocation et la définition des fonctions des membres des organes de la SE qu'il prévoit, suggèrent l'idée d'une responsabilité sociale à la base de la notion de gouvernance. Les opérations suivantes requièrent l'autorisation de l'organe de surveillance ou une délibération de l'organe d'administration :

tout projet d'investissement dont le volume est supérieur à un pourcentage donné du capital souscrit, la création, l'acquisition, l'aliénation ou la liquidation d'entreprises, d'établissements ou de parties d'établissements, lorsque le prix de rachat ou le produit de la cession est supérieur à un pourcentage déterminé du capital souscrit,

tout recours au crédit ou l'octroi de crédits, l'émission d'obligations et la reprise ou le cautionnement d'engagements de tiers, lorsque l'opération globale est supérieure à un pourcentage déterminé du capital souscrit,

la passation de contrats de ventes et de prestation de services lorsque le chiffre d'affaires global prévu est supérieur à un pourcentage donné du chiffre d'affaires du dernier exercice commercial,

35 Le conseil d'administration dans le système moniste doit comporter en principe au moins trois membres- article 43.2 du règlement ou la durée de leur mandat : "les membres des organes de la société sont nommés pour une période fixée par les statuts, qui ne peut excéder six ans ».

36 Article 39.1 dans le système dualiste et article 43.1 dans le système moniste.

37 J.L. Colombani et M. Favero, Societas Europea, « Pratiques et Affaires 2001 », Paris 2002.p. 53. 
Le pourcentage visé aux tirets susmentionnés est fixé par les statuts. Il ne peut être inférieur à 5\% ni supérieur à $25 \%$.

Le système de gouvernance (moniste ou dualiste) est choisi par l'assemblée générale des actionnaires. La décision est inscrite dans les statuts de la SE et est exclue des négociations entre direction et représentation des salariés. Ainsi, les salariés n'ont officiellement pas voix au chapitre quant au choix du système de gouvernance (et ne peuvent donc pas décider d'être par la suite représentés au conseil d'administration ou de surveillance). Que ce soit dans le système moniste ou dualiste, la représentation du personnel ne peut pas empêcher qu'une décision du conseil d'administration ou de surveillance soit prise si les représentants des actionnaires adoptent une position commune ${ }^{38}$. Les statuts de la $\mathrm{SE}$ énumèrent les catégories d'opérations qui donnent lieu à autorisation préalable du conseil de surveillance (système dualiste) ou à décision expresse du conseil d'administration (système moniste). Dans certains Etats membres, l'organe de surveillance peut soumettre lui-même à autorisation certaines opérations. Par ailleurs, les Etats membres peuvent établir la liste de ces opérations pour les SE immatriculées sur leur territoire ${ }^{39}$. Les droits concrets dont jouissent le conseil d'administration et le conseil de surveillance varient donc largement en fonction du pays dans lequel la SE est immatriculée et des statuts de cette dernière. Les représentants des salariés au conseil d'administration ou de surveillance ont les mêmes de droits et obligations que les représentants des actionnaires, y compris le droit de vote et le droit à rémunération. Tous les membres des organes de la SE sont soumis à une obligation de confidentialité ; ils ne sont pas autorisés à divulguer des informations susceptibles de porter préjudice aux intérêts de la société ${ }^{40}$.

\section{La situation des salariés}

Dans le cadre de ses travaux sur les SE, le réseau SEEurope a établi différents types de $\mathrm{SE}$ afin de mieux décrire une diversité, qui a évolué rapidement depuis octobre $2004^{41}$. Cette classification permet de distinguer les SE menant une activité et employant des salariés $(S E$ « normales »), des $\mathrm{SE}$ menant une activité, mais n'employant pas de salariés

38 Règlement relatif à la SE, art. 50.

39 Règlement relatif à la SE, art. 48.

40 Règlement relatif àla SE, art. 49.

41 Le Réseau SEEurope - organisé par l'Institut syndical européen - a été fondée en 2003 pour observer la transposition de la législation SE et son impact pratique sur les entreprises et les relations industrielles. Le réseau implique des spécialistes des relations juridiques, économiques et industrielles de l'Espace économique européen (États membres de l'UE plus la Norvège, l'Islande et Lichtenstein). Au fil des ans, le réseau a produit un large éventail de publications, y compris les rapports nationaux, des études de cas et des rapports thématiques. Dès le début, la recherche et la surveillance a été menée en vue de répondre également aux besoins des praticiens en Europe impliqués dans la création d'une SE. 
$(S E$ « vides ») et des SE ne menant aucune activité et n'employant aucun salarié ( $S E$ « coquilles vides » $)^{42}$. Les SE qui sont probablement opérationnelles, mais pour lesquelles nous ne disposons pas d'informations suffisantes pour procéder à leur classification (par exemple, dont nous ne savons pas si elles emploient effectivement des salariés) sont classées dans la catégorie SE OVNI (Objets Volants Non Identifiés).

La plupart des SE normales ont été constitués par voie de fusion entre deux (ou plusieurs) sociétés ou par la transformation d'une société anonyme existante. Un nouveau mode de constitution de facto a cependant fait son apparition au fil du temps: l'activation d'une $\mathrm{SE}$ « coquille vide ». Une $\mathrm{SE}$ « coquille vide » activée est transformée en SE « vide » en démarrant une activité économique (sans l'embauche de salariés) ou en $\mathrm{SE}$ « normale » en démarrant une activités économique et en transférant des salariés dans la société ${ }^{43}$. Un marché pour ce type de « coquilles vides » s'est développé, notamment en Allemagne et en République Tchèque. Ce type de SE sert souvent d'instrument de constitution d'autres $\mathrm{SE}$ coquilles vides (sous forme de filiales) qui sont ensuite vendues à des clients intéressés. Le nouvel acquéreur active la SE en y transférant des salariés et/ ou en démarrant une activité .

Dans la pratique, la majorité des $\mathrm{SE}$ « normales » ont conservé leur système de gouvernance d'origine. Un pourcentage important de $\mathrm{SE}$ « normales », notamment en Allemagne, a toutefois opté pour un conseil unique, forme de gouvernance qui n'existait pas jusqu'à présent dans le système allemand ${ }^{44}$.

\section{La SE dans l'espace européen}

Il faut souligner que la localisation de la SE dans l'espace européen soulève beaucoup d'enjeux qu'ils soient fiscaux, de gouvernance ou statutaires.

Le règlement du Conseil CE n²157/2001 du 8 octobre 2001 reste muet quant au régime fiscal. Depuis, la directive CE n ${ }^{\circ}$ 2005/91 du 17 février 2005, modifiant la directive CEE n ${ }^{\circ}$ 90/434 du 23 juillet 1990 concernant « le régime fiscal commun applicable aux fusions, apports d'actifs et échanges d'actions intéressant des sociétés d'Etats membres différents ", a consacré la neutralité fiscale du transfert de siège de la SE vers un autre Etat membre. Si la constitution de la SE bénéficie de mesures fisacles favorables, il n'en va pas encore de même au cours de sa vie sociale, où elle relève de la fiscalité des Etats membres où elle est implantée. Le transfert de siège de la SE a été cependant organisé pour être effectué en franchise d'impôt ; ce qui peut permettre d'ores et déjà une certaine optimisation fiscale. Enfin, une avancée significative en faveur de la SE sera accomplie par la mise en

42 M. Stollt, E. Wolters, Implication des travailleurs dans la Société européenne (SE). Guide pour les acteurs de terrain, Bruxelles 2013, p. 458.

43 Ibidem, p. 477.

44 Ibidem, p. 482. 
place d'une " assiette commune consolidée de l'impôt sur les sociétés ", dite " ACCIS ", à laquelle les services de la Commission travaillent actuellement ${ }^{45}$. La directive du 23 juillet 1990, modifiée par la directive du 17 février 2005, a vocation à s'appliquer aux opérations de fusion, d'apport d'actifs ou d'échange d'actions contribuant à la constitution d'une SE issue d'une fusion ou d'un apport d'actifs transfrontalier. Ce règime commun, tel que transposé dans les droits nationaux, assure en principe une neutralité fiscale en matière d'impôt sur le revenu des opérations de fusion, d'apport d'actifs et d'échange de titres ${ }^{46}$. Deux séries de dispositions prévoient, d'une part, une exonération des plus-values d'apport réalisées par les sociétés fusionnées ou les sociétés apporteuses (l'apport de branche d'activité ou apport de titres permettant d'acquérir la majorité des droits de vote de la société qui reçoit cet apport), sous certaines conditions et avec certains aménagements, et, d'autre part, un régime de sursis d'imposition permettant aux actionnaires des sociétés absorbées de différer, sous certaines conditions et limites, l'imposition de plus-values d'échange de leurs titres ${ }^{47}$.

En France, la neutralité fiscale prévue par la directive est organisée selon un régime dit de " faveur » par l'article $210 \mathrm{~A}$ du Code général des impôts (CGI) pour les opérations de fusion, et par l'article $210 \mathrm{~B}$ dudit code pour les apports partiels d'actifs et assimilés. En cas d'apport, l'engagement de conserver pendant au moins trois ans les titres reçus par la société apporteuse doit être souscrit, ou repris par certains actionnaires, de la société apporteuse ayant procédé, dans le cadre de l'article 115.2 du CGI, à une répartition des titres reçus en rémunération de l'apport. En cela, les opérations de constitution d'une SE ne sont pas traitées différemment de celles réalisées par d'autres sociétés fusionnant dans un cadre transfrontalier européen et supportent donc les mêmes difficultés et contraintes fiscales nationales dans la mise en oeuvre de la directive, y compris en termes de procédure. Ainsi, ce régime de faveur exige un agrément préalable en cas de fusion-absorbation ou d'apport partiel d'actifs d'une société française par une société d'un autre Etat. Cet agrément est en principe accordé de droit lorsque l'opération est

45 ACCIS (l'assiette commune consolidée pour l'impôt sur les sociétés), est un ensemble unique de règles de détermination du résultat imposable, susceplibles dêtre utilisées par les sociétés exerçant leur activité au sein de l'UE. En d'autres termes, une société ou un groupe de sociétés éligible ne devrait se conformer qu'à un seul régime au sein de l'Union pour calculer son résultat imposable, plutôt qu'aux différents régimes propres à chacun des Etats membres dans lesquels l'activité est exercée. En outre, les groupes soumis au régime ACCIS auraient la possibilité de ne remplir qu'une seule déclaration fiscale consolidée pour l'ensemble de leurs activités au sein de l'UE. Les résultats imposables consolidés du groupe seraient répartis entre chacune des sociétés qui le constituent par application d'une formule simple permettant à chaque Etat membre d'imposer les bénéfices des sociétés résidentes de cet Etat, au taux d'imposition choisi par celui-ci (comme c'est le cas aujourd'bui).

46 N. Lenoir, P.P. Bruneau et M. Menjucq, Les enjeux de la localisation de la SE dans l'espace européen, « Droit \&Patrimoine », n 163, Octobre 2007, p. 89.

47 Ibidem, p. 95. 
justifiée par un motif économique, et n'a pas comme objectif principal ou un de ses objectifs principaux la fraude ou l'évasion fiscale ; les modalités de l'opération permettent l'imposition future des plus-values placées en sursis d'imposition ${ }^{48}$. Cependant, cet agrément impose que l'opération se traduise par la constitution d'un établissement stable de la société absorbante en France, reprenant à son bilan l'ensemble des élements d'actifs de la société française absorbée, et est subordonné à la souscription des engagements prévus par l'article $210 \mathrm{~A}$ du CGI, qui doivent être en fait respectés par cet établissement stable. La SE ne bénéficie, en cours de vie, d'aucun régime fiscal spécifique défini au niveau européen, et relève donc des diffèrents systèmes fiscaux des Etats membres, en accord, le cas échéant, avec les conventions fiscales applicables. Elle bénéficie du même régîme fiscal que les sociétés anonymes. Les établissements stables qui sont généralement créés dans les différents Etats membres à la suite de la constitution d'une SE relèvent des fiscalités nationales des Etats d'implantation et doivent donc déterminer leurs résultats imposables sur une base territoriale, le cas échént dans vingt-sept Etats différents si la SE est implantée dans chacun des Etats membres. Pour l'instant, la SE n'offre donc aucune possibilité de détermination globale de son résultat imposable et d'allocation de ce résultat imposable entre les Etats membres. Cette situation réduit les avantages attachés à la simplification des structures de la $\mathrm{SE}$, qui permettent une organisation juridique et comptable unifiée au niveau européen. La création de SE pose naturellement la question de la localisation et du transfert de son siège statutaire, notamment pour optimiser son régime fiscal, en tirant profit de la concurrence fiscale. Notons cependant que le transfert de siège implique également le transfert de son administration centrale ; le siège d'une $\mathrm{SE}$ et son administration centrale devant être réunis dans le même Etat membre; ce qui réduit le risque de délocalisation à but purement fiscal. Le règlement du Conseil $\mathrm{CE}$ $n^{\circ}$ 2157/2001 permettant à la SE de transférer son siège statutaire, dans un autre Etat membre sans qu'il en résulte ni dissolution, ni création d'une personne morale nouvelle, la directive du 17 février 2005 a naturellement étendu le régime fiscal commun de neutralité au transfert du siège statutaire de la SE vers un autre Etat membre. Ce transfert n'entraîne donc pas en principe d'imposition des plus-values qui seraient généralement dues par suite de la dissolution de la société transférant son siège, sous la réseve, déjà mentionnée, du rattachement des actifs ainsi transférés à un établissement stable dans l'Etat de départ ; ce qui rend possible, en cas de cession, l'imposition ultérieure des plus-values. Lorsque le pays de départ ne soumet pas à l'impôt les titres de participation, et n'impose donc pas le maintien d'un établissement stable, le transfert du siège statutaire d'une SE n'ayant qu'une activité de holding aura donc une pleine portée tant juridique que fiscale. L'Autriche paraît être le seul Etat membre a avoir autoriser la mise en oeuvre de la rentabilité fiscale des opérations transfrontalières, sans la subordonner au maintien,

48 Art. 210.3 B CGI. 
d'un établissement stable dans l'Etat d'origine. Il en va autrement lorsque le pays de départ impose le maintien d'un établissement stable en cas de transfert du siège d'une holding. Ainsi, la France a aménagé son droit interne pour permettre le transfert vers un autre Etat membre du siège d'une société française à la condition du maintien d'un établissement stable ${ }^{49}$. Ce transfert peut donc désormais intervenir selon un régime de neutralité fiscale ; l'imposition, le cas échéant, des plus-values liées aux actifs de la SE étant reportée sur l'établissement stable dont elle dispose en France à la suite du transfert de siège. Depuis le 1er janvier 2007, les plus-values à long terme réalisées sur les cessions de titres de participation sont imposables au taux de $0 \%$; seule une quote-part égale à 5 $\%$ des plus-values nettes de cession étant imposable à l'impôt sur les sociétés au taux de droit commun ${ }^{50}$. Dans le cas où la société française absorbée est une pure société holding, qui ne détient que des titres de participation, la question de l'exigence du maintien d'un établissement stable en France s'est donc posée à compter du 1er janvier 2007 ; cette exigence pouvant apparaître comme excessive au regard de l'évolution du droit européen. Pour assurer pleinement la mobilité de la SE, la solution serait de supprimer l'exigence du maintien de l'établissement stable pour les holdings qui transfèrent leur siège.

Le principe de la taxation des plus-values sur les actifs cédés, attribuables à l'Etat d'origine, après le transfert du siège serait maintenu ; son application effective pouvant être assurée par un corps de contrôle spécialement créé à cet effet au niveau européen. A n'en pas douter, les SE tireraient alors pleinement parti des différences importantes entre les régimes d'impôt sur les sociétés existant dans l'Union. Mais on peut aussi penser qu'un mouvement de transfert de siège de SE vers les Etats fiscalement plus cléments verrait le jour, nonobstant l'exigence du maintien de l'établissement stable dans le pays d'origine et l'obligation de se soumettre à la fiscalité propre à chacun des Etats d'implantation. Au moins la SE bénéficiera-t-elle du régime d'imposition du pays d'accueil pour ses nouvelles activités. La logique de la $\mathrm{SE}$ voudrait qu'elle soit soumise à un statut fiscal propre, dont le Parlement européen a souligné l'importance dès $2001^{51}$.

La solution envisagée par la Commision et le groupe de travail qu'elle a constitué en 2004 était l'adoption d'une ACCIS pour les SE. La SE souscrirait alors, dans l'Etat de son siège, une seule déclaration d'impôt sur les sociétés faisant état de l'ensemble de son résultat net imposable, correspondant à l'ACCIS, réalisé tant dans l'Etat où est situé son siège que par les établissements et les filiales dont elle possède la totalité du capital, situés dans d'autres Etats membres. Un seul corps de règles s'appliquerait donc à la détermina-

\section{Art. 221.2 CGI.}

50 Ces plus-values sont ainsi imposées à un taux effectif de 1,72\%.

51 Communication Commission au Conseil, 23 octobre 2001: " la totalité des avantages découlant de la société européenne ne se concrétiseront que si les entreprises peuvent opter pour cette structure sans encourir des coûts fiscaux additionnels et si certains obstacles fiscaux actuels entravant l'extension des activités à plus d'un Etat membre sont supprimés ». 
tion de l'assiette imposable, mettant ainsi fin, sur ce plan, à une « concurrence fiscale dommageable $\aleph^{52}$. Le règime de l'ACCIS pourrait cependant être optionnel. Deux méthodes d'imposition seraient alors envisageables : soit l'assujettisement de l'ACCIS à un impôt de type fédéral, perçu au niveau de l'Union, soit la répartition de l'ACCIS entre les différents Etats où la SE dispose d'établissements stables, ou de filiales, selon une clé dont les termes restent à définir ; chacun des Etats fixant lui-même son taux d'imposition. On peut actuellement penser que les travaux de la Commission sorientent vers cette seconde solution. Une certaine concurrence fiscale serait alors maintenue. Comme l'indiquait la Commission, " le succès du statut de la SE apparaît comme étroitement lié à la mise en place d'un régime commun d'imposition des sociétés ${ }^{33}$ ». Bien que non limité à la SE, l'ACCIS est un élément déterminant et nécessaire pour le succès de celle-ci. On peut penser que sa mise en place; souhaitée par les milieux d'affaires; bénéficierait en effet pleinement à la SE, en permettant à cette structure unique de réduire ses coûts de gestion, tout en préservant une certaine concurrence fiscale. La position des Etats membres sur le projet d'ACCIS n'est cependant pas unanime. Mais il faut être clair : si l'on veut encourager les $\mathrm{SE}$ à développer leurs activités transfrontalières, alors même qu'en l'état, les questions fiscales relèvent de la règle de l'unanimité au sein de l'Union, il faut arriver à une nouvelle directive fiscale intégrant l'ACCIS.

La marge d'autonomie statutaire de la SE dépend de la législation de l'Etat de son siège statutaire. L'existence d'enjeux statutaires pourrait étonner à l'égard de la SE, qui, afin de conserver une unité de son statut indépendamment de la localisation de son siège social dans tel ou tel État membre, devrait limiter les possibilités pour ses actionnaires de modifier le corpus juridique prévu par le règlement portant statut de la SE précitée ${ }^{54}$.

Les options laissées aux États membres dans les matières couvertes par le règlement du Conseil CE n 2157/2001 sont nombreuses et d'importance. Sans prétendre à l'exhaustivité, il est possible de citer quelques-unes des options relatives à l'organisation interne de la SE à prendre en considération lors du choix de sa localisation. Ainsi, dans les SE dualistes, l'article 39 paragraphe 2, prévoit que le ou les membres de l'organe de direction sont nommés et révoqués par l'organe de surveillance, mais que l'État membre du siège de la SE peut prévoir ou donner aux statuts la possibilité que le ou les membres de l'organe de direction soient nommés et révoqués par l'assemblée générale des actionnaires dans les mêmes conditions que pour une SA locale. La France a levé partiellement cette option car la révocation des membres du directoire par l'assemblée générale est

52 Il existe déjà un mécanisme européen concernant la concurrence fiscale dommageable, mais qui reste très partiel, fondé sur le Code de bonne conduite dans le domaine de la fiscalité des entreprises adopté par le Conseil ECOFIN du 2 décembre 1997, et dont le suivi est assuré par un groupe de travail placé auprès de la Commission.

53 Communication de la Commmission au Conseil, 23 octobre 2001.

54 N. Lenoir, P.P. Bruneau et M. Menjucq, Les enjeux de la localisation de la SE dans l'espace européen, « Droit \&Patrimoine ", n 163, Octobre 2007, p. 98. 
incluse dans le droit interne des SA, au contraire du Royaume-Uni qui n'a nullement levé l'option à défaut de connaître une structure dualiste pour ses sociétés par actions. En outre, pour les deux systèmes, le règlement du Conseil $\mathrm{CE} \mathrm{n}{ }^{\circ} 12572001$ énonce que les statuts fixent le nombre de membres des différents organes d'administration, de direction et de surveillance mais que les États membres peuvent déterminer un maximum et/ ou un minimum. La France a levé cette option par référence aux dispositions françaises sur les SA, le Royaume-Uni et la Belgique n'ayant fixé qu'un nombre minimum, mais pas de maximum.

Les dispositions nationales ont une importance d'autant plus grandes qu'elles comblent les nombreuses lacunes du règlement du Conseil $\mathrm{CE} \mathrm{n}{ }^{\circ}$ 2157/2001 concernant le statut de la SE qui est éloigné de la forme rêvée dans les années soixante-dix d'une société de pur droit européen. La hiérarchique des normes nationales est définie par l'article L. 229-1 du Code du commerce, qui précise que la SE est d'abord régie par le règlement du Conseil $\mathrm{CE} \mathrm{n}^{\circ}$ 2157/2001, puis par les dispositions du chapitre IX du Code du commerce, ainsi que par les dispositions applicables aux SA dans la mesure où elles ne sont pas contraires aux dispositions spécifiques aux SE. Il résulte de cette multiplicité de normes applicables que lors de la domiciliation du siège de la $\mathrm{SE}$, il n'est pas inutile de consulter le droit local des SA qui s'applique aussi à la $\mathrm{SE}$ dans le silence dudit règlement et de règles nationales spécifiques. À cet égard, la domiciliation du siège de la SE dans un État membre ayant un droit des SA peu rigide entraîne nécessairement un assouplissement du statut de la SE. Ainsi, compte tenu de la souplesse du droit anglais des sociétés par actions, une SE domiciliée au Royaume-Uni devrait globalement disposer d'une plus grande liberté statutaire qu'une SE immatriculée en Belgique.

La SE, se rattachant à un modèle institutionnel plus que concractuel de la société commerciale, ne dispose pas à première vue d'une grande liberté statutaire. Il est plus exact de dire que l'étendue de cette liberté varie selon la législation des Etats membres. Certes l'article 9 du règlement du Conseil CE n² 2157/2001 précise que la hiérarchie des normes applicables à la $\mathrm{SE}$, se réfère aux statuts de chaque société immédiatement après ses propres dispositions. Mais le même article prévoit par ailleurs qu'il revient aux législations nationales de préciser les possibilités offertes aux associés et actionnaires d'adapter, de compléter ou de modifier les règles de droit européen ou national régissant la gouvernance de la SE. Pour les matières traitées par le règlement, la SE n'est tout d'abord régie qur par ses statuts que lorsque ledit règlement l'autorise expressément ${ }^{55}$. Et ce, sous réserve encore que l'Etat membre où la société a son siège statutaire, n'ait pas adopté de dispositions contraires. C'est ainsi que, si le paragraphe 1er de l'article $48 \mathrm{du}$ règlement du Conseil $\mathrm{CE} \mathrm{n}^{\circ}$ 2157/2001 confère aux statuts le soin de définir les « catégories d'opérations qui donnent lieu à autorisation de l'organe de surveillance, dans le système

55 Art. 9.1 du règlement relatif à la $\mathrm{SE}$. 
dualiste, ou à décision expresse de l'organe d'administration, dans le système moniste ", le paragraphe 3 attribue au contraire compétence aux Etats membres de fixer, s'ils le souhaitent, les catégories d'opérations en question ${ }^{56}$. Tel est le choix qu'a fait le législateur français en transposant à la $\mathrm{SE}$ les dispositions applicables aux SA sur les conventions réglementées. Celles-ci sont soumises à l'approbation, non pas de l'organe d'administration ou de surveillance, mais de l'assemblée générale des actionnaires. La levée de cette option a été vivement critiquée par les milieux d'affaires français qui ont fait valoir que dans les autres Etats membres les modalités d'approbation des conventions réglementées étaient généralement définies par les statuts. Cet exemple illustre bien la nature du compromis ayant permis à la SE de voir le jour : les Etats se sont mis d'accord sur l'institution d'une Societas Europea à la condition que son statut ne s'éloigne pas trop de leurs conceptions à la fois politiques et juridiques.

Le rôle des Etats dans la définition des règles de gouvernance de la SE est encore plus accentué s'agissant des matières non couvertes (ou couvertes partiellement) par le règlement. Dans cette hypothèse, la $\mathrm{SE}$ voit sa liberté statutaire soumise aux mêmes conditions que celles imposées par le droit de l'Etat membre où elle a son siège statutaire en ce qui concerne les sociétés anonymes ${ }^{57}$. Il en résulte qu'une $\mathrm{SE}$ immatriculée au Royaume-Uni par exemple aura une marge d'autonomie statutaire sensiblement plus étendue qu'une SE immatriculée en Allemagne. Ceci vaut pour une série de dispositions concernant notamment le nombre ou les modalités de nomination ou de révocation des membres des organes de surveillance, de direction ou d'administration, ou encore la possibilité pour des personnes morales d'être membres d'un de ces organes. Cette dernière possibilité, qui n'existe pas pour les SA en France et en Allemagne, n'a pas été ouverte dans le cadre de la SE, alors que, traditionnellement reconnue au Royaume-Uni pour les sociétés anonymes, elle vaut aussi pour les SE. Donc, en général, parmi les motivations $\mathrm{du}$ recours au statut de la SE figure le souci d'alléger les contraintes de la régulation propre au secteur financier. Cette préocupation est même parfois présentée, par certains dirigeants, comme l'une des justifications essentielles de constitution en SE.

\section{Les conclusions : les avantages et les perspectives}

La réglementation du droit spécial des sociétés s’étoffe de jours en jours. Le législateur a introduit des réformes presque tous les semestres, voir tous les trimestres.. La matière est évolutive. La production normative en ce domaine donne elle aussi lieu à un véritable

56 H. Vanoorbeek, La société européenne et la strategie de léentreprise dans le contexte européen, Paris 2009, p.449

57 J.L. Colombani et M. Favero, Societas Europea, « Pratiques et Affaires 2001 », Paris 2002. p. 58. 
" flux normatif tendu ", en droit interne, comme en droit de l'Union, comme d'ailleurs en matière de sociétés de capitaux.

Premierement, c'est ainsi que la société européenne, dont la personnalité juridique n'est donc pas nationale à proprement parler, est néanmoins une société anonyme régie par le droit national du pays où est implanté son siège. De plus, la $\mathrm{SE}$ répond au besoin des entreprises de faciliter leurs activités au sein de l'espace européen car l'existence de formes de sociétés commerciales différentes pouvait créer certains freins. Lobjectif premier était de créer une forme régie par les mêmes règles quelque soit l'Etat membre d'immatriculation. Mais cela n'a pas été possible car chaque Etat membre tirait vers ses spécificités. La SE est un compromis avec des bases communes, mais aussi des renvois au droit national de la sociéé anonyme de l'Etat membre d'immatriculation.

Il faut ajouter que les salariés sont plutôt rassurés par la création d'une société européenne puisque cela leur donne droit à l'application d'un système de cogestion. Dans la $\mathrm{SE}$, les salariés sont obliagtoirement représentés au sein de la direction.

De plus, cela rend, ainsi, possible une croissance externe et une réorganisation des entreprises dont l'activité n'est pas limitée à une satisfaction de besoins purement locaux. La SE se présente donc comme le moyen le moins cher et le plus simple pour organiser la coopération, l'intégration ou la restructuration d'entreprises d'Etat membres différents.

On peut souligner aussi, que la création de sociétés à la dimension du marché interne où la constitution de sociétés dont la formation et le fonctionnement sont régis par un règlement du droit européen, à côté de sociétés relevant d'un droit national, contribuent à faire correspondre, dans toute la mesure du possible, l'unité économique et l'unité juridique de l'entreprise européenne. La SE est le seul moyen de transférer le siège social dans un autre Etat membre sans dissolution ni création d'une personne morale nouvelle et à la simple majorité qualifiée des actionnaires alors que dans la plupart des Etats membres, l'unanimité est de rigueur.

Le statut de SE pourrait amener à une espèce de law shopping qui consisterait à mettre les droits nationaux des sociétés en concurrence. Les $\mathrm{SE}$ s'implanteraient là où le droit des sociétés est le plus accueillant. À defaut d'harmonisation européenne, c'est donc le droit de l'Etat membre dans lequel la $\mathrm{SE}$ a son siège, qui a vocation à être appliqué et qui peut être plus ou moins contraignant. Ce genre de forum shopping est régulièrement hors de portée des sociétés qui ne sont pas déjà de véritables acteurs européens et habituées à manier des systèmes juridiques différents.

La possibilité de transfert du siège statutaire dans un autre pays présente un avantage important pesant en faveur du choix de la SE. Avec ce régime juridique, les sociétés peuvent passer plus facilement d'un État membre à un autre. La SE a grandement simplifié les fusions transfrontalières par rapport aux règles et législations nationales complexes préexistantes. 
On peut supposer que toute entreprise envisageant d'établir une SE conduit une analyse profit et perte. La contribution de la $\mathrm{SE}$ à une réduction substantielle des coûts ne peut pas être généralisée. Il est cependant possible que la SE permette de réaliser des économies, par exemple, en entraînant une rationalisation des structures de gestion et la dissolution de certaines de ses filiales nationales.

Enfin, il faut ajouter que la régulation relative à la $\mathrm{SE}$ donne la possibilité de créer une société privée européenne (SPE). Cette nouvelle forme juridique est encore en cours d'élaboration et fait débat parmi les États membres. La SPE est prévue principalement pour les petites et moyennes entreprises, mais rien n'empêcherait les grandes entreprises d'adopter ce statut (en lieu et place de la $S E$ ). Du point de vue du salariat, on peut craindre qu'il soit utilisé pour échapper aux règles nationales sur la participation des salariés.

\section{SUMMARY}

\section{The European company: issues and prospects}

The article is an explanation of the way the European company operates. The author briefly points to the laws and their provisions regulating the status of the European company in its present form. The author primarily highlights EU legislation in the field of European company law, but also regulations in French commercial law related to the analyzed company. Additionally, the author shows the ways of creating a European company and possible styles of managing it. The author also highlights the situation of the employees of a European company and the advantages of creating this type of company, whether fiscal, related to the location, or resultant from the statute of this company. In conclusion, the author points out a number of prospects for the functioning of a European company, highlighting the competitiveness of such a company on the European market and the possibility of its development by European enterprises, as well as indicating that the company is a kind of compromise between the regulations proposed by the European legislator and the regulations laid down by the domestic law of the Member States.

KEYwords: European company, European Union law, commercial law 
La société européene... $\mid 223$ 
